\title{
The Continuity of Fuzzy Metric Spaces
}

\author{
Raghad I. Sabri*
}

Applied Science Department, University of Technology, Baghdad, Iraq. E-mail: 100247@uotehnology.edu.iq

Abstract: In the present paper, the definitions of a fuzzy continuous function and uniformly fuzzy continuous are introduced. We prove that a function $\mathrm{f}$ from a fuzzy metric space $\left(\widetilde{\mathrm{F}}, \widetilde{\mathcal{M}}_{\widetilde{\mathrm{F}}}\right)$ into a fuzzy metric space $\left(\widetilde{\mathrm{G}}, \widetilde{\mathcal{M}}_{\widetilde{\mathrm{G}}}\right)$ is fuzzy continuous if and only if for every fuzzy open subset $\widetilde{A}$ of $\widetilde{G}, f^{-1}(\widetilde{A})$ is fuzzy open in $\widetilde{F}$. Also the composition function of two uniformly fuzzy continuous functions is proved to be a uniformly fuzzy continuous function.

Keywords: Fuzzy Metric Space; Fuzzy Continuous Function; Uniformly Fuzzy Continuous Function

\section{Introduction}

The foundation of the concept of the fuzzy metrics is given for the first time by Kramosil and Michalek in $1975^{[1]}$ to enforce the fuzziness principle to the traditional concepts of metric and metric spaces to introduce the definition of the fuzzy metrics space by generalizing the notion of the probabilistic metric space to the fuzzy case. Kaleva and Seikkala in $1984^{[2]}$ generalized the idea of a metric space to introduce the definition of the fuzzy metrics space by specifying the distance between two points (rather than the probabilistic metric space) to be a non-negative fuzzy number. Sostak in ${ }^{[3]}$ presented an alternative method to introduce a new version of the concept of a fuzzy metric called revised fuzzy metric. A $\mathrm{t}$-conorm binary operation is used in the definition of the revised fuzzy metric to assess the degree of proximity of two points. Moreover, many researchers had different views on the problem of constructing a fuzzy metric space. In particular, the concept of fuzzy metric space given in ${ }^{[1]}$ is modified by George and Veeramani ${ }^{[4]}$ in terms of Hausdorff topology. In addition, Xie et al. ${ }^{[5]}$ studied the relation between the fuzzy measure and the fuzzy metric space. Authors in ${ }^{[6]}$ gave a new definition of fuzzy metric space by using fuzzy scalars instead of fuzzy numbers or real numbers and proved basic theories about this space. $\operatorname{In}^{[7]}$ Gupta and Kanwar have made efforts to introduce V-fuzzy metric spaces and to study their main properties. Other notations and approaches for fuzzy metric spaces are considered in ${ }^{[8-11]}$.

The aim of this paper is to introduce the definition of a fuzzy continuous function and uniformly fuzzy continuous function in a fuzzy metric space (FM-space) $(\widetilde{\mathrm{F}}, \tilde{\mathcal{M}})$ given in ${ }^{[12]}$ and proved essentialy theorems.

Several researcher can calculate the properties of the materials in applied physics using mathematical models by means of these theorems ${ }^{[13-91]}$.

The structure of this paper is as follows. In section 2 some properties and basic notions of the fuzzy metric space (FM-space) are given. The concepts of a fuzzy continuous and uniformly fuzzy continuous function are introduced in section 3 , moreover, some important properties of the given definitions are investigated.

\section{Preliminaries}

Copyright (C) 2020 Raghad I. Sabri

doi: 10.18686/esta.v7i4.132

This is an open-access article distributed under the terms of the Creative Commons Attribution Non-Commercial License

(http://creativecommons.org/licenses/by-nc/4.0/), which permits unrestricted non-commercial use, distribution, and reproduction in any medium, provided the original work is properly cited. 
In this section, we restate basic results and some definitions.

\section{Definition 2.1 ${ }^{[92]}$}

A fuzzy metric space (briefly, FM-space) is an ordered pair $(\tilde{\mathrm{F}}, \tilde{\mathcal{M}})$ where $\tilde{\mathrm{F}}$ is a fuzzy set and $\tilde{\mathcal{M}}$ is a mapping from $\tilde{\mathrm{F}} \times \tilde{\mathrm{F}} \times(0,1]$ into $\mathrm{I}=[0,1]$ such that the following five properties hold, for each $(\mathrm{a}, \alpha),(\mathrm{b}, \beta),(\mathrm{c}, \delta) \in \tilde{\mathrm{F}}$ :

$(\tilde{\mathcal{M}} 1) \tilde{\mathcal{M}}(\mathrm{a}, \mathrm{b}, \gamma)>0$ if $\mathrm{a} \neq \mathrm{b}$ where $\gamma=\max \{\alpha, \beta\}$

$(\tilde{\mathcal{M}} 2) \tilde{\mathcal{M}}(\mathrm{a}, \mathrm{b}, \gamma)=0$ if and only if $\mathrm{a}=\mathrm{b}$.

( $\widetilde{\mathcal{M}} 3) \tilde{\mathcal{M}}(\mathrm{a}, \mathrm{b}, \gamma)=\widetilde{\mathcal{M}}(\mathrm{b}, \mathrm{a}, \gamma)$.

$(\tilde{\mathcal{M}} 4) \tilde{\mathcal{M}}(\mathrm{a}, \mathrm{b}, \gamma) \leq \tilde{\mathcal{M}}(\mathrm{a}, \mathrm{c}, \gamma)+\tilde{\mathcal{M}}(\mathrm{c}, \mathrm{b}, \gamma)$, where $\gamma=\max \{\alpha, \beta, \delta\}$

$(\tilde{\mathcal{M}} 5)$ If $0<\lambda \leq \gamma<1$ then $\tilde{\mathcal{M}}(\mathrm{a}, 0, \gamma) \leq \tilde{\mathcal{M}}(\mathrm{a}, 0, \lambda)$ and there exists $0<\gamma_{\mathrm{n}}<\gamma$ such that $\lim _{n \rightarrow \infty} \tilde{\mathcal{M}}\left(a_{n}, 0, \gamma_{n}\right)=\tilde{\mathcal{M}}(a, 0, \gamma)$.

Definition 2.2 ${ }^{[92]}$

Let $(\widetilde{\mathrm{F}}, \widetilde{\mathcal{M}})$ be an FM-space, and let $(\mathrm{a}, \alpha) \in \widetilde{\mathrm{F}}$, where $\alpha \in(0,1]$. Given real number $\varepsilon>0$, then:

(1) $\widetilde{\mathrm{O}}_{\varepsilon}\left(\mathrm{a}_{1}, \alpha_{1}\right)=\left\{(\mathrm{a}, \alpha) \in \tilde{\mathrm{F}}: \tilde{\mathcal{M}}\left(\mathrm{a}, \mathrm{a}_{1}, \gamma\right)<\varepsilon\right\}$ is called the fuzzy open ball of radius $\varepsilon$ where $\gamma=\max \left\{\alpha, \alpha_{1} \in(0,1]\right\}$.

(2) $\widetilde{\mathrm{B}}_{\varepsilon}\left[\mathrm{a}_{1}, \alpha_{1}\right]=\left\{(\mathrm{a}, \alpha) \in \tilde{\mathrm{F}}: \tilde{\mathcal{M}}\left(\mathrm{a}, \mathrm{a}_{1}, \gamma\right) \leq \varepsilon\right\}$ is called the fuzzy closed ball of radius $\varepsilon$.

Definition 2.3 ${ }^{[92]}$

Let $(\tilde{\mathrm{F}}, \tilde{\mathcal{M}})$ be an FM-space. A fuzzy subset $\widetilde{\mathrm{A}} \subseteq \tilde{\mathrm{F}}$ is fuzzy open if and only if there exists a fuzzy open ball $\widetilde{\mathrm{O}}_{\varepsilon}(\mathrm{a}, \alpha)$ centered at every fuzzy point $(\mathrm{a}, \alpha)$ in $\widetilde{\mathrm{A}}$ that are contained in $\widetilde{\mathrm{A}}$. A fuzzy subset $\widetilde{\mathrm{B}} \subseteq \widetilde{\mathrm{F}}$ is called fuzzy closed if $\widetilde{B}^{c}=\widetilde{F}-\widetilde{B}$ is fuzzy open.

Definition 2.4 ${ }^{[92]}$

In an FM-space $(\tilde{\mathrm{F}}, \tilde{\mathcal{M}})$, a fuzzy sequence $\left\{\left(a_{n}, a_{n}\right)\right\}$ where $a, a_{n} \in(0,1]$ is said to be

(i) Convergent if there exists $(\mathrm{a}, a) \in \tilde{\mathrm{F}}$ such that $\lim _{n \rightarrow \infty} \tilde{\mathcal{M}}\left(a_{n}, a, \gamma\right)=0$ where $\gamma=\max \left\{a_{n}, a\right\}$ or simply written $\left\{\left(a_{n}, a_{n}\right)\right\} \rightarrow(a, a)$.

(ii) Cauchy if for all $\varepsilon>0$ there is an integer number $\mathrm{N} \in \mathbb{N}$ such that $\tilde{\mathcal{M}}\left(\mathrm{a}_{\mathrm{n}}, \mathrm{a}_{\mathrm{m}}, \gamma\right)<\varepsilon$ for every $\mathrm{n}$, $\mathrm{m} \geq \mathrm{N}$ where $\gamma=\max \left\{\alpha_{\mathrm{n}}, \alpha_{\mathrm{m}}\right\}$.

Definition 2.5 ${ }^{[92]}$

A fuzzy set $\widetilde{A}$ in an FM-space $(\widetilde{\mathrm{F}}, \widetilde{\mathcal{M}})$ is called fuzzy bounded if there exists $0<\mathrm{r}<1$ such that $\tilde{\mathcal{M}}(\mathrm{a}, \mathrm{b}, \gamma)<\mathrm{r} . r$ call for each $(\mathrm{a}, \alpha),(\mathrm{b}, \beta) \in \tilde{\mathrm{F}}, \gamma$ $=\max \{\alpha, \beta\}$.

Definition 2.6 ${ }^{[92]}$

A fuzzy sequence $\left\{\left(a_{n}, a_{n}\right)\right\}$ in an FM-space
$(\widetilde{\mathrm{F}}, \widetilde{\mathcal{M}})$ is said to be fuzzy bounded if the corresponding fuzzy set is fuzzy bounded.

Definition 2.7 ${ }^{[92]}$

An FM-space $(\widetilde{\mathrm{F}}, \tilde{\mathcal{M}})$ is called complete if every Cauchy fuzzy sequence in $\widetilde{\mathrm{F}}$ is a fuzzy convergent.

Definition 2.8 ${ }^{[92]}$

Let $(\tilde{\mathrm{F}}, \tilde{\mathcal{M}})$ be an FM-space and let $\left\{\left(a_{n}, a_{n}\right)\right\}_{n \geq 1}$ be a fuzzy sequence of real numbers. Given $r_{1}<r_{2}<\cdots<r_{n} \ldots$ be strictly increasing sequence of natural numbers. Then $\left\{\left(a_{n k}, a_{n k}\right)\right\}_{n \geq 1}$ is called a fuzzy subsequence of $\left\{\left(a_{n}, \propto_{n}\right)\right\}_{n \geq 1}$.

Definition 2.9 ${ }^{[12]}$

Let $\mathrm{U}$ be a universal set, then for any $\alpha \in(0,1]$ and $\mathrm{u} \in \mathrm{U}$, a fuzzy subset $\mathrm{u}_{\mathrm{a}}$ of $\mathrm{U}$ is called a fuzzy point in $U$ if

$\mathrm{u}_{\alpha}(\mathrm{w})=\left\{\begin{array}{c}\alpha \text { if } \mathrm{u}=\mathrm{w} \\ 0 \text { otherwise }\end{array}\right.$ for each $w \in U$.

Now, the definition of a fuzzy metric space is given.

\section{Fuzzy continuous and uniformly fuzzy continuous function on fuzzy metric space}

In this section, the continuity of the fuzzy metric space is discussed. So the definition of the fuzzy continuous function at a fuzzy point in the fuzzy metric space is introduced initially.

\section{Definition 3.1}

Let $\left(\widetilde{\mathrm{F}}, \widetilde{\mathcal{M}}_{\widetilde{\mathrm{F}}}\right)$ and $\left(\widetilde{\mathrm{G}}, \widetilde{\mathcal{M}}_{\widetilde{\mathrm{G}}}\right)$ be an FM-spaces and let $\widetilde{A} \subseteq \widetilde{F}$. Then the function $f: \widetilde{A} \rightarrow \widetilde{G}$ is said to be fuzzy continuous function at a fuzzy point $(a, \alpha) \in \widetilde{A}$ if for every $\varepsilon>0$ there exists $\sigma>0$ such that $\widetilde{\mathcal{M}}_{\widetilde{\mathrm{G}}}(\mathrm{f}(\mathrm{b}), \mathrm{f}(\mathrm{a}), \gamma)<\varepsilon \quad$ whenever $(\mathrm{b}, \beta) \in \widetilde{\mathrm{A}}$ implies $\widetilde{\mathcal{M}}_{\widetilde{\mathrm{F}}}(\mathrm{b}, \mathrm{a}, \gamma)<\sigma$.

The next theorem gives a characterization of the fuzzy continuous function.

\section{Theorem 3.2}

Suppose that $\left(\tilde{\mathrm{F}}, \widetilde{\mathcal{M}}_{\widetilde{\mathrm{F}}}\right)$ and $\left(\widetilde{\mathrm{G}}, \widetilde{\mathcal{M}}_{\widetilde{\mathrm{G}}}\right)$ be two FM-spaces and let $\widetilde{A} \subseteq \widetilde{F}$. The function $f: \widetilde{A} \rightarrow \widetilde{G}$ is a fuzzy continuous function at a fuzzy point $(a, \alpha) \in \widetilde{A}$ if and only if the sequence $\left\{\left(f\left(b_{n}, \alpha_{n}\right)\right)\right\}$ fuzzy converges to $f(a, \alpha)$ for any fuzzy sequence of fuzzy points $\left\{\left(b_{n}, \alpha_{n}\right)\right\}$ in $\widetilde{A}$ that fuzzy converges to $(a, \alpha)$.

Proof

Assume that the function $f: \tilde{A} \rightarrow \tilde{G}$ is fuzzy continuous at a fuzzy point $(a, \alpha)$ in $\tilde{A}$. Let $\left\{\left(b_{n}, \alpha_{n}\right)\right\}$ be a 
fuzzy sequence in $\tilde{A}$ that is fuzzy converges to $(a, \alpha)$. Given $\varepsilon>0$ and by the continuity of $f$ there is $\sigma>0$ such that $\tilde{\mathcal{M}}_{\tilde{G}}(f(b), f(a), \gamma)<\varepsilon$ whenever $(b, \beta) \in \tilde{A}$ satisfy $\tilde{\mathcal{M}}_{\tilde{F}}(b, a, \gamma)<\sigma$. Since the fuzzy sequence $\left\{\left(b_{n}, \alpha_{n}\right)\right\}$ converges to $(a, \alpha)$ then we can find a number $n \geq N$ with $\tilde{\mathcal{M}}_{\tilde{F}}\left(b_{\mathrm{n}}, a, \gamma\right)<\sigma$. Hence for $n \geq N$ implies $\tilde{\mathcal{M}}_{\tilde{G}}\left(f\left(b_{n}\right), f(a), \gamma\right)<\varepsilon \quad$, where $\quad \gamma=$ $\max \left\{\alpha_{n}, \alpha \in(0,1]\right\}$. Thus the fuzzy sequence $\left\{\left(f\left(b_{n}, \alpha_{n}\right)\right)\right\}$ fuzzy converges to $f(a, \alpha)$. For the converse, assume that every fuzzy sequence $\left\{\left(b_{n}, \alpha_{n}\right)\right\}$ in $\tilde{A}$ converging to $(a, \alpha)$ has the property that the fuzzy sequence $\left\{\left(f\left(b_{n}, \alpha_{n}\right)\right)\right\}$ fuzzy converges to $f(a, \alpha)$. We shall prove that $f$ is fuzzy continuous at $(a, \alpha)$. We claim $f$ is not fuzzy continuous at $(a, \alpha)$. Then there exists some $\varepsilon>0$ and for which no $\sigma>0$ can satisfy the requirement that $(b, \beta) \in \tilde{A}$ and $\tilde{\mathcal{M}}_{\tilde{F}}(b, a, \gamma)<\sigma$ implies $\tilde{\mathcal{M}}_{\tilde{G}}(f(b), f(a), \gamma)<\varepsilon$, that means for each $\sigma>0$ there is a fuzzy point $(b, \beta) \in \tilde{A}$ with $\tilde{\mathcal{M}}_{\tilde{F}}(b, a, \gamma)<\sigma$ but $\tilde{\mathcal{M}}_{\tilde{G}}(f(b), f(a), \gamma) \geq \varepsilon$. Now for each $n \in N$, there is a fuzzy point $\left(b_{n}, \alpha_{n}\right) \in \tilde{A}$ with $\tilde{\mathcal{M}}_{\tilde{F}}\left(b_{n}, a, \gamma\right) \leq\left(1-\frac{1}{n}\right)$ but $\tilde{\mathcal{M}}_{\tilde{G}}\left(f\left(b_{n}\right), f(a), \gamma\right) \geq \varepsilon$. Then the fuzzy sequence $\left\{\left(b_{n}, \alpha_{n}\right)\right\}$ fuzzy converges to fuzzy point $(a, \alpha)$ but the fuzzy sequence $\left\{\left(f\left(b_{n}, \alpha_{n}\right)\right)\right\}$ does not fuzzy converges to $f(a, \alpha)$. This contradicts the assumption that each sequence $\left\{\left(b_{n}, \alpha_{n}\right)\right\}$ in $\tilde{A}$ fuzzy converging to $(a, \alpha)$ with the property $\left\{\left(f\left(b_{n}, \alpha_{n}\right)\right)\right\}$ fuzzy converging to $f(a, \alpha)$. Hence our claim that $f$ is not fuzzy continuous at $(a, \alpha)$ must be false.

One more characterization for the fuzzy continuous function is assigned in the following result.

\section{Theorem 3.3}

Let $\left(\tilde{F}, \tilde{\mathcal{M}}_{\tilde{F}}\right)$ and $\left(\tilde{G}, \tilde{\mathcal{M}}_{\tilde{G}}\right)$ be two FM-spaces. A function $f: \tilde{F} \rightarrow \tilde{G}$ is fuzzy continuous at $(a, \alpha) \in \tilde{F}$ if and only if for every $\varepsilon>0$ there exists $\sigma>0$ such that $\tilde{O}_{\sigma}(a, \alpha) \subseteq f^{-1}\left[\tilde{O}_{\varepsilon}(a, \alpha)\right]$.

\section{Proof}

The function $f: \tilde{F} \rightarrow \tilde{G}$ is fuzzy continuous at $(a, \alpha) \in \tilde{F}$ if and only if for every $\varepsilon>0$ there exists $\sigma>0 \quad$ with $\tilde{\mathcal{M}}_{\tilde{G}}(f(b), f(a), \gamma)<\varepsilon$ for each $(b, \beta) \in \tilde{F}$ implies $\widetilde{\mathcal{M}}_{\tilde{F}}(b, a, \gamma)<\sigma$ and this mean $(b, \beta) \in \tilde{O}_{\sigma}(a, \alpha)$ satisfying $f(b, \beta) \in \tilde{O}_{\varepsilon}(f(a, \alpha))$ or $f\left[\tilde{O}_{\sigma}(a, \alpha)\right] \subseteq \tilde{O}_{\varepsilon}(f(a, \alpha)) \quad$. Hence $\quad \tilde{O}_{\sigma}(a, \alpha) \subseteq$ $f^{-1}\left[\tilde{O}_{\varepsilon}(a, \alpha)\right]$.

According to the previous theorem, the following corollary is proved.

\section{Corollary 3.4}

A function $f: \tilde{F} \rightarrow \tilde{G}$ is fuzzy continuous on $\tilde{F}$ if and only if for every fuzzy open subset $\tilde{A}$ of $\tilde{G}$, $f^{-1}(\tilde{A})$ is fuzzy open in $\tilde{F}$ where $\left(\tilde{F}, \tilde{\mathcal{M}}_{\tilde{F}}\right)$ and $\left(\tilde{G}, \tilde{\mathcal{M}}_{\tilde{G}}\right)$ are FM-spaces.

\section{Proof}

Assume that $f$ is a fuzzy continuous function and let $\tilde{A}$ be a fuzzy open set in $\tilde{G}$. We prove that $f^{-1}(\tilde{A})$ is fuzzy open in $\tilde{F}$. Since $\emptyset$ and $\tilde{F}$ are fuzzy open, we may assume that $f^{-1}(\tilde{A}) \neq \varnothing$ and $f^{-1}(\tilde{A}) \neq \tilde{F}$. Consider $(b, \beta) \in f^{-1}(\tilde{A})$ then $f(b, \beta) \in \tilde{A}$. By assumption, $\tilde{A}$ is fuzzy open so there is and may assume that $\mathrm{n}$ ubset $\mathrm{t}$ $\varepsilon>0$ such that $\tilde{O}_{\varepsilon}(f(b, \beta)) \subseteq \tilde{A}$, since $f$ is fuzzy continuous at $(b, \beta)$ by Theorem (3.3) there is some $\sigma>0 \quad$ with $\quad \tilde{O}_{\sigma}(b, \beta) \subseteq f^{-1}\left[\tilde{O}_{\varepsilon}(b, \beta)\right] \subseteq f^{-1}(\tilde{A})$. Hence each fuzzy point $(b, \beta)$ of $f^{-1}(\tilde{A})$ is an interior fuzzy point and so $f^{-1}(\tilde{A})$ is a fuzzy open in $\tilde{F}$.

Conversely, suppose that $f^{-1}(\tilde{A})$ is a fuzzy open in $\tilde{F}$ for any fuzzy open set $\tilde{A}$ of $\tilde{G}$. Now for each $\varepsilon>0$, the fuzzy ball $\tilde{O}_{\varepsilon}(f(b, \beta))$ where $(b, \beta) \in \tilde{F}$ is fuzzy open in $\tilde{F}$. Since $(b, \beta) \in f^{-1}\left[\tilde{O}_{\varepsilon}(b, \beta)\right]$ its follows that there is some $\sigma>0$ with $\tilde{O}_{\sigma}(b, \beta) \subseteq$ $f^{-1}\left[\tilde{O}_{\varepsilon}(b, \beta)\right]$ and by Theorem (3.3) concludes that $f$ is fuzzy continuous.

\section{Corollary 3.5}

Let $\left(\tilde{F}, \tilde{\mathcal{M}}_{\tilde{F}}\right)$ and $\left(\tilde{G}, \tilde{\mathcal{M}}_{\tilde{G}}\right)$ be an FM-spaces. A function $f: \tilde{F} \rightarrow \tilde{G}$ is fuzzy continuous on $\tilde{F}$ if and only if $f^{-1}(\tilde{A})$ is fuzzy closed in $\tilde{F}$ for each fuzzy closed subset $\tilde{A}$ of $\tilde{G}$.

\section{Proof}

Consider $\tilde{A}$ be a fuzzy closed subset of $\tilde{G}$ then $\tilde{G}-\tilde{A}$ is fuzzy open in $\tilde{G}$ therefore $f^{-1}(\tilde{G}-\tilde{A})$ is fuzzy open in $\tilde{F}$ by Corollary (4.4). But $f^{-1}(\tilde{G}-\tilde{A})=$ $\tilde{F}-f^{-1}(\tilde{A})$ so $f^{-1}(\tilde{A})$ is fuzzy closed in $\tilde{F}$.

Conversely, assume that $f^{-1}(\tilde{A})$ is fuzzy closed in $\tilde{F}$ for each fuzzy closed subset $\tilde{A}$ of $\tilde{G}$. But the whole space $\tilde{F}$ and $\emptyset$ are fuzzy closed set, then $\tilde{F}-f^{-1}(\tilde{A})$ is fuzzy open in $\tilde{F}$ and $f^{-1}(\tilde{G}-\tilde{A})=\tilde{F}-f^{-1}(\tilde{A})$ is fuzzy open in $\tilde{F}$. Since each fuzzy open subset of $\tilde{G}$ is of the type $\tilde{G}-\tilde{A}$, where $\tilde{A}$ is a fuzzy closed set and by using Corollary (3.4) it follows that $f$ is fuzzy continuous.

The following theorem demonstrates the composition function of two fuzzy continuous functions must be a fuzzy continuous function.

\section{Proposition 3.6}

Let $\left(\tilde{F}, \tilde{\mathcal{M}}_{\tilde{F}}\right),\left(\tilde{G}, \tilde{\mathcal{M}}_{\tilde{G}}\right)$ and $\left(\tilde{P}, \tilde{\mathcal{M}}_{\tilde{P}}\right)$ be three 
FM-spaces and let $f: \tilde{F} \rightarrow \tilde{G}, h: \tilde{G} \rightarrow \tilde{P}$ be fuzzy continuous functions. Then the composition function $(h \circ f): \widetilde{F} \rightarrow \tilde{P}$ is a fuzzy continuous function.

\section{Proof}

Let $\tilde{A}$ be a fuzzy open subset of $\tilde{P}$. By Corollary (4.4) $h^{-1}(\tilde{A})$ is a fuzzy open subset of $\tilde{G}$. Again by Corollary (3.4), we get $f^{-1}\left(h^{-1}(\tilde{A})\right)$ is a fuzzy open subset of $\tilde{F}$, since $(h \circ f)^{-1}(\tilde{A})=f^{-1}\left(h^{-1}(\tilde{A})\right)$ and from Corollary (3.4) we conclude that $(h \circ f)$ is a fuzzy continuous function.

The concept of uniformly fuzzy continuous function in an FM-space is introduced in the following definition.

\section{Definition 3.7}

Let $\left(\tilde{F}, \tilde{\mathcal{M}}_{\tilde{F}}\right)$ and $\left(\tilde{G}, \tilde{\mathcal{M}}_{\tilde{G}}\right)$ be two FM-spaces. A function $f: \tilde{F} \rightarrow \tilde{G}$ is said to be uniformly fuzzy continuous on $\tilde{F}$ if for each $\varepsilon>0$ there is some $\sigma>0$ such that $\quad \tilde{\mathcal{M}}_{\tilde{G}}\left(f\left(a_{1}\right), f\left(a_{2}\right), \gamma\right)<\varepsilon \quad$ whenever $\tilde{\mathcal{M}}_{\tilde{F}}\left(a_{1}, a_{2}, \gamma\right)<\sigma$.

The Cauchy fuzzy property of the sequence in an FM-space discusses in the following theorem.

\section{Theorem 3.8}

Suppose that $\left(\tilde{F}, \tilde{\mathcal{M}}_{\tilde{F}}\right)$ and $\left(\tilde{G}, \tilde{\mathcal{M}}_{\tilde{G}}\right)$ are two FM-spaces and let $f: \tilde{F} \rightarrow \tilde{G}$ be a uniformly fuzzy continuous function. If $\left\{\left(a_{n}, \alpha_{n}\right)\right\}$ is a Cauchy fuzzy sequence in $\tilde{F}$ then $\left\{\left(f\left(a_{n}, \alpha_{n}\right)\right)\right\}$ is Cauchy fuzzy sequence in $\tilde{G}$.

\section{Proof}

Let $f$ be uniformly fuzzy continuous. Then by definition(3.7) for each $\varepsilon>0$ there is $\sigma>0$ such that $\tilde{\mathcal{M}}_{\tilde{G}}\left(f\left(a_{1}\right), f\left(a_{2}\right), \gamma\right)<\varepsilon \quad$ whenever $\tilde{\mathcal{M}}_{\tilde{F}}\left(a_{1}, a_{2}, \gamma\right)<$ $\sigma$, for each $\left(a_{1}, \alpha_{1}\right),\left(a_{2}, \alpha_{2}\right) \in \tilde{F}$, where $\gamma=$ $\max \left\{\alpha_{1}, \alpha_{2} \in(0,1]\right\}$. Since $\left\{\left(a_{n}, \alpha_{n}\right)\right\}$ is a Cauchy fuzzy sequence in $\tilde{F}$ corresponding to $\sigma>0$ there is an integer number $N \in \mathbb{N}$ with $\tilde{\mathcal{M}}_{\tilde{F}}\left(a_{n}, a_{m}, \gamma\right)<\sigma$ for any $m, n \geq N$. Hence we conclude that $\tilde{\mathcal{M}}_{\tilde{G}}\left(f\left(a_{n}\right), f\left(a_{m}\right), \gamma\right)<\varepsilon$ for each $m, n \geq N$ and this implies that $\left\{\left(f\left(a_{n}, \alpha_{n}\right)\right)\right\}$ is a Cauchy fuzzy sequence.

The following theorem demonstrates the composition function of two uniformly fuzzy continuous functions must be a uniformly fuzzy continuous function.

\section{Proposition 3.9}

Let $\left(\tilde{F}, \tilde{\mathcal{M}}_{\tilde{F}}\right),\left(\tilde{G}, \tilde{\mathcal{M}}_{\tilde{G}}\right)$ and $\left(\tilde{P}, \tilde{\mathcal{M}}_{\tilde{P}}\right)$ be an FM-spaces. Let $f: \tilde{F} \rightarrow \tilde{G}$ and $h: \tilde{G} \rightarrow \tilde{P}$. Then if $f$ is uniformly fuzzy continuous on $\tilde{F}$ and $h$ is uniformly fuzzy continuous on $f(\tilde{F})$ then $(h \circ f): \tilde{F} \rightarrow \tilde{P}$ is uniformly fuzzy continuous on $\tilde{F}$.

\section{Proof}

Let $\varepsilon>0$ be given. We want to find a $\sigma>0$ such that if $\left(a_{1}, \alpha_{1}\right),\left(a_{2}, \alpha_{2}\right) \in \tilde{F}$ and $\tilde{\mathcal{M}}_{\tilde{F}}\left(a_{1}, a_{2}, \gamma\right)<$ $\sigma$ then $\tilde{\mathcal{M}}_{\tilde{P}}\left(h\left(f\left(a_{1}\right)\right), h\left(f\left(a_{2}\right)\right), \gamma\right)<\varepsilon$. Since $h$ is uniformly fuzzy continuous on $f(\tilde{F})$ then there exists a $\sigma_{1}>0$ such that if $f\left(a_{1}, \alpha_{1}\right), f\left(a_{2}, \alpha_{2}\right) \in \tilde{F}$ and $\tilde{\mathcal{M}}_{\tilde{G}}\left(f\left(a_{1}\right), f\left(a_{2}\right), \gamma\right)<\sigma_{1}$.

Then,

$$
\tilde{\mathcal{M}}_{\tilde{P}}\left(h\left(f\left(a_{1}\right)\right), h\left(f\left(a_{2}\right)\right), \gamma\right)<\varepsilon
$$

Now, since $f$ is uniformly fuzzy continuous on $\tilde{F}$ then there exists a $\sigma_{2}>0$ such that if $\left(a_{1}, \alpha_{1}\right),\left(a_{2}, \alpha_{2}\right) \in \tilde{F}$ and $\tilde{\mathcal{M}}_{\tilde{F}}\left(a_{1}, a_{2}, \gamma\right)<\sigma_{2}$.

Then, $\quad \tilde{\mathcal{M}}_{\tilde{G}}\left(f\left(a_{1}\right), f\left(a_{2}\right), \gamma\right)<\sigma_{1}$

Put $\sigma=\sigma_{2}$, then for each $\left(a_{1}, \alpha_{1}\right),\left(a_{2}, \alpha_{2}\right) \in \tilde{F}$ we have that (1) holds. Since (1) holds we have that (2) holds. Thus $(h \circ f)$ is uniformly fuzzy continuous on $\tilde{F}$.

\section{Conclusion}

In this paper, the definitions of fuzzy continuous function from fuzzy metric space $\left(\tilde{F}, \widetilde{\mathcal{M}}_{\tilde{F}}\right)$ into a fuzzy metric space $\left(\tilde{G}, \tilde{\mathcal{M}}_{\tilde{G}}\right)$ is introduced then several properties are discussed. Also the definition of uniformly fuzzy continuous function from a fuzzy metric space $\left(\tilde{F}, \tilde{\mathcal{M}}_{\tilde{F}}\right)$ into a fuzzy metric space $\left(\tilde{G}, \widetilde{\mathcal{M}}_{\tilde{G}}\right)$ is given and essential theorems are proved.

\section{References}

1. Kramosil I, Michalek J. Fuzzy metrics and statistical metric spaces. Kybernetika 1975; 11(5): 336344.

2. Kaleva O, Seikkala S. On fuzzy metric spaces. Fuzzy Sets and Systems 1984; 12(3): 215-229.

3. Sostak A. George-Veeramani fuzzy metrics revised. Axioms 2018; 7(60): 1-6.

4. George A, Veeramani P. On some results in fuzzy metric spaces. Fuzzy Sets Systems 1994; 64(3): 395-399.

5. Xie J, Li Q, Chen S, et al. The fuzzy metric space based on fuzzy measure. Open Mathematics 2016; 14(1): 603-612.

6. Xia Z, Guo F. Fuzzy metric spaces. Journal of Applied Mathematics and Computing 2004; 16(1-2): 371-381.

7. Gupta V, Kanwar A. V-Fuzzy metric space and related fixed point theorems. Fixed Point Theory and Applications 2016; 2016(1): 1-17.

8. Ali MNM, Sadk F, Sabre R. Applications of fixed fuzzy point theorem. Al-Mustansiriyah Journal of Science 2015; 26(2): 57-61. 
9. Omran S, Al-Saadi HS. Some notes on metric and fuzzy metric spaces. International Journal of Advanced and Applied Sciences 2017; 4(5): 41-43.

10. Rashid $\mathrm{MH}$. Some results on fuzzy metric spaces. International Journal of Open Problems in Computer Science and Mathematics 2016; 9(4): 1-23.

11. Beg I, Sedghi S, Shobe N. Fixed point theorems in fuzzy metric spaces. International Journal of Analysis 2013; 2013: 1-4.

12. Sabre RI, Ali MNM. New fuzzy metric spaces and fuzzy matrices. Journal of the College of Basic Education 2014; 20(82): 1-6.

13. Shihab SN, Sarhan MA. Convergence analysis of shifted fourth kind chebyshev wavelets. IOSR Journal of Mathematics 2014; 10(2): 54-58.

14. Al-Rawi SN. Numerical solution of integral equations using Taylor series. Journal of the College of Education 1998; 5: 51-60.

15. Shihab SN, Naif TN. On orthonormal Bernstein polynomial of order eight. Open Science Journal of Mathematics and Application 2014; 2(2): 15-19.

16. Delphi M, Shihab S. State parametrization basic spline functions for trajectory optimization. The Journal of Natural Sciences, Life and Applied Sciences 2019; 3(4): 110-119.

17. Delphi M, Shihab S. Modified basic spline, multiscaling and wavelets algorithms for optimal control problems [master's thesis]. Iraq: University of Technology; 2019.

18. Shihab SN, Naif TN. Direct methods based on orthonormal Bernstein polynomials for solving optimal control problems [master's thesis]. Iraq: University of Technology; 2014.

19. Shihab SN, Sarhan MA. Numerical solution of variational calculus using chebyshev wavelets method [PhD thesis]. Iraq: University of Technology; 2015.

20. Shihab SN, Falah AH. Efficient numerical algorithms for the optimal control problem [master's thesis]. Iraq: University of Technology; 2008.

21. Shihab SN, Al-Janabi ASAR. On the numerical solution for solving some continuous optimal control problems [PhD thesis]. Iraq: Al-Mustansiriyah University; 2005.

22. Al-Faour O, Shihab SN, Al-Saleni BF. Multistep methods for solving nonlinear integral equations. Journal of the College of Basic Education 2001; 12(2).

23. Al-Faour O, Shihab SN, Al-Nasser RH. Expansion method for solving volterra equations. Journal of Babylon University 2000; 7(3): 1355-1362.

24. Al-Faour O, Al-Ani FID, Al-Rawi SN. Numerical evaluation of fourier transformation using orthogonal functions. Engineering and Technology Journal 2000; 17(7).

25. Al-Ani FID, Al-Faour O, Al-Rawi SN. Numerical solution of variational calculus using chebyshev wavelet method. Journal of Al-rafidain University 1997; 2: 1-10.

26. Shihab S, Delphi M. Direct iterative algorithm for solving optimal control problems using B-Spline polynomials. Emirates Journal for Engineering Research 2019; 24(4): 1-9.

27. Alrawy SS, Salih AA. Shifted modified chebyshev direct method for solving quadratic optimal control problem. Samarra Journal of Pure and Applied Science 2020; 2(1): 67-75.

28. Shihab S, Ali HA, Kasheem BE, et al. Legendre wavelets method for solving boundary value problems. Journal of the College of Basic Education 2012; 18(76): 73-86.

29. Shihab SN, Asmaa AA, Ali MNM. Collocation orthonormal Bernstein polynomials method for solving integral equations. Engineering and Technology Journal 2015; 33(8 Part (B)): 1493-1502.

30. Delphi M, Shihab S. Modified iterative algorithm for solving optimal control problems. Open Science Journal of Statistics and Application 2019; 6(2): 20-27.

31. Al-Rawi SN. On the solution of certain fractional integral equations. Journal of Kirkuk University Scientific Studies 2006; 1(2).

32. Shihab SN, Abdalrehman AA. Numerical solution of calculus of variations by using the second chebyshev wavelets. Engineering and Technology Journal 2012; 30(18): 3219-3229.

33. Shihab SN, Abdelrehman AA. Some new relationships between the derivatives of first and second chebyshev wavelets. International Journal of Engineering, Business and Enterprise Application 2012.

34. Shehab SN, Ali HA, Yaseen HM. Least squares method for solving integral equations with multiple time lags. Engineering and Technology Journal 2010; 28(10): 1893-1899.

35. Shihab SN, Abdalrehman AA. Solving optimal control linear systems by using new third kind chebyshev wavelets operational matrix of derivative. Baghdad Science Journal 2014; 11(2): 229-234.

36. Delphi M, Shihab S. Operational matrix basic spline wavelets of derivative for linear optimal control problem. Electronics Science Technology and Application 2019; 6(2): 18-24.

37. Shihab SN, Sarhan MA. New operational matrices of shifted fourth chebyshev wavelets. Elixir International Journal-Applied Mathematics 2014; 69: 23239-23244.

38. Rasheed MS. Study of the effects of acidic solutions on the physical properties of polymeric materials superimposed. Al-Mustansiriyah Journal of Science 2012; 13(49): 6.

39. Rasheed MS, Mahde HS. Electronic combination lock design using remote control. Journal of the College of Basic Education 2012; 18(75): 265-280.

40. Rasheed MS, Mohammed AN. Design of a laser based free space communication system: Laser, optical communication, free space. LAP LAMBERT Academic Publishing 2012.

41. Rasheed MS. Approximate solutions of barker equation in parabolic orbits. Engineering and Technology Journal 2010; 28(3): 492-499.

42. Rasheed MS. An improved algorithm for the solu- 
tion of Kepler's equation for an elliptical orbit. Engineering and Technology Journal 2010; 28(7): 1316-1320.

43. Rasheed MS. Acceleration of predictor-corrector Halley method in astrophysics application. International Journal of Emerging Technologies in Computational and Applied Sciences 2012; 1(2): 91-94.

44. Rasheed MS. A fast procedure for solving two-body problem in celestial mechanic. International Journal of Engineering, Business and Enterprise Applications 2012; 1(2): 60-63.

45. Rasheed MS. Solve the position to time equation for an object travelling on a parabolic orbit in celestial mechanics. Diyala Journal for Pure Science 2013; 9(4): 37-47.

46. Rasheed MS. Comparison of starting values for implicit iterative solutions to hyperbolic orbits equation. International Journal of Software and Web Sciences 2012; 1(2): 65-71.

47. Rasheed MS. On solving hyperbolic trajectory using new predictor-corrector quadrature algorithms. Baghdad Science Journal 2014; 11(1): 186-192.

48. Rasheed MS. Modification of three order methods for solving satellite orbital equation in elliptical motion. Journal of University of Anbar for Pure Science 2020; 14(1): 33-37.

49. Rasheed M, Barille R. Room temperature deposition of $\mathrm{ZnO}$ and $\mathrm{Al}: \mathrm{ZnO}$ ultrathin films on glass and PET substrates by DC sputtering technique. Optical and Quantum Electronics 2017; 49(5).

50. Rasheed M, Barille R. Optical constants of DC sputtering derived ITO, $\mathrm{TiO}_{2}$ and $\mathrm{TiO}_{2}: \mathrm{Nb}$ thin films characterized by spectrophotometry and spectroscopic ellipsometry for optoelectronic devices. Journal of Non-Crystalline Solids 2017; 476: 1-14.

51. Rasheed M, Barille R. Comparison the optical properties for $\mathrm{Bi}_{2} \mathrm{O}_{3}$ and $\mathrm{NiO}$ ultrathin films deposited on different substrates by DC sputtering technique for transparent electronics. Journal of Alloys and Compounds 2017; 728: 1186-1198.

52. Saidani T, Zaabat M, Aida MS, et al. Influence of precursor source on sol-gel deposited $\mathrm{ZnO}$ thin films properties. Journal of Materials Science: Materials in Electronics 2017; 28(13): 9252-9257.

53. Boumezoued A, Guergouri K, Barille R, et al. ZnO nanopowders doped with bismuth oxide, from synthesis to electrical application. Journal of Alloys and Compounds 2019; 791: 550-558.

54. Bouras D, Mecif A, Barille $\mathrm{R}$, et al. $\mathrm{Cu}: \mathrm{ZnO}$ deposited on porous ceramic substrates by a simple thermal method for photocatalytic application. Ceramics International 2018; 44(17): 21546-21555.

55. Saidi W, Hfaidh N, Rasheed M, et al. Effect of $\mathrm{B}_{2} \mathrm{O}_{3}$ addition on optical and structural properties of $\mathrm{TiO}_{2}$ as a new blocking layer for multiple dye sensitive solar cell application (DSSC). RSC Advances 2016; 6(73): 68819-68826.

56. Auktuolis A, Girtan M, Mousdis GA, et al. Measurement of charge carrier mobility in perovskite nanowire films by photo-celiv method. Proceedings of the Romanian Academy-Series A: Mathematics, Physics, Technical Sciences, Information Science 2017; 18(1): 34-41.

57. Dkhilalli F, Megdiche S, Guidara K, et al. AC conductivity evolution in bulk and grain boundary response of sodium tungstate $\mathrm{Na}_{2} \mathrm{WO}_{4}$. Ionics 2018; 24(1): 169-180.

58. Dkhilalli F, Borchani SM, Rasheed M, et al. Structural, dielectric, and optical properties of the zinc tungstate $\mathrm{ZnWO}_{4}$ compound. Journal of Materials Science: Materials in Electronics 2018; 29(8): 6297-6307.

59. Dkhilalli F, Borchani SM, Rasheed M, et al. Characterizations and morphology of sodium tungstate particles. Royal Society Open Science 2018; 5(8): 172214.

60. Enneffati M, Louati B, Guidara K, et al. Crystal structure characterization and AC electrical conduction behavior of sodium cadmium orthophosphate. Journal of Materials Science: Materials in Electronics 2018; 29(1): 171-179.

61. Kadri E, Krichen M, Mohammed R, et al. Electrical transport mechanisms in amorphous silicon/crystalline silicon germanium heterojunction solar cell: Impact of passivation layer in conversion efficiency. Optical and Quantum Electronics 2016; 48(12).

62. Kadri E, Messaoudi O, Krichen M, et al. Optical and electrical properties of $\mathrm{SiGe} / \mathrm{Si}$ solar cell heterostructures: Ellipsometric study. Journal of Alloys and Compounds 2017; 721: 779-783.

63. Kadri E, Dhahri K, Zaafouri A, et al. AC conductivity and dielectric behavior of a $-\mathrm{Si}: \mathrm{H} / \mathrm{c}-\mathrm{Si}_{1-\mathrm{y}} \mathrm{Ge}_{\mathrm{y}}$ $/ \mathrm{p}-\mathrm{Si}$ thin films synthesized by molecular beam epitaxial method. Journal of Alloys and Compounds 2017; 705: 708-713.

64. Azaza NB, Elleuch S, Rasheed M, et al. 3-(p-nitrophenyl) coumarin derivatives: Synthesis, linear and nonlinear optical properties. Optical Materials 2019; 96: 109328.

65. Enneffati M, Rasheed M, Louati B, et al. Morphology, UV-visible and ellipsometric studies of sodium lithium orthovanadate. Optical and Quantum Electronics 2019; 51(9).

66. Rasheed M, Sarhan MA. Solve and implement the main equations of photovoltaic cell parameters using visual studio program. Insight-Mathematics 2019; 1(1): 18-26.

67. Rasheed M, Sarhan MA. Characteristics of solar cell outdoor measurements using fuzzy logic method. Insight-Mathematics 2019; 1(1): 1-8.

68. Rasheed M, Sarhan MA. Measuring the solar cell parameters using fuzzy set technique. Insight-Electronic 2019; 1(1): 1-9.

69. Rasheed M. Linear programming for solving solar cell parameters. Insight-Electronic 2019; 1(1): 1016.

70. Rasheed M. Investigation of solar cell factors using fuzzy set technique. Insight-Electronic 2019; 1(1): $17-23$. 
71. Rasheed M, Barille R. Development and characterization of single and multilayer thin films for optoelectronics application [PhD thesis]. France: University of Angers; 2017.

72. Rasheed M, Shihab S. Analytical modeling of solar cells. Insight-Electronic 2019; 1(2): 1-9.

73. Rasheed M, Shihab S. Modeling and simulation of solar cell mathematical model parameters determination based on different methods. Insight-Mathematics 2019; 1(1): 1-16.

74. Rasheed M, Shihab S. Parameters estimation for mathematical model of solar cell. Electronics Science Technology and Application 2019; 6(1): 2028.

75. Ouda EH, Shihab S, Rasheed M. Boubaker wavelet functions for solving higher order integro-differential equations. Journal of Southwest Jiaotong University 2020; 55(2).

76. Sarhan MA, Shihab S, Rasheed M. A new boubaker wavelets operational matrix of integration. Journal of Southwest Jiaotong University 2020; 55(2).

77. Abdulrahman AA, Rasheed M, Shihab S. Discrete hermite wavelet filters with prove mathematical aspects. Journal of Southwest Jiaotong University 2020; 55(2).

78. Rasheed M, Abdulrahman AA, Shihab S. Discrete chebyshev wavelet transformation with image processing. Journal of Southwest Jiaotong University 2020; 55(2)

79. Mitlif RJ, Rasheed M, Shihab S. An optimal algorithm for fuzzy transportation problem. Journal of Southwest Jiaotong University 2020; 55(3).

80. Sarhan MA, Shihab S, Rasheed M. On the properties of two-dimensional normalized boubaker polynomials. Journal of Southwest Jiaotong University 2020; 55(3).

81. Aziz SH, Rasheed M, Shihab S. New properties of modified second kind chebyshev polynomials. Journal of Southwest Jiaotong University 2020;
55(3).

82. Mitlif RJ, Rasheed M, Shihab S. An optimal algorithm for fuzzy transportation problem. Journal of Southwest Jiaotong University 2020; 55(3).

83. Kashem BE, Ouda EH, Aziz SH, et al. Some results for orthonormal boubaker polynomials with application. Journal of Southwest Jiaotong University 2020; 55(3).

84. Mohammedali MN, Sabri RI, Rasheed M, et al. Some results on G-Normed linear spaces. Journal of Southwest Jiaotong University 2020; 55(3).

85. Sabri RI, Mohammedali MN, Rasheed M, et al. Compactness of soft fuzzy metric space. Journal of Southwest Jiaotong University 2020; 55(3).

86. Rasheed M, Shihab S. Numerical techniques for solving parameters of solar cell. Applied Physics 2020; 3(2): 15-27.

87. Nema M, Gupta L, Trivedi NR. Video compression using SPIHT and SWT wavelet. International Journal of Electronics and Communication Engineering 2012; 5(1): 1-8.

88. Pandian R, Vigneswara T, Kumari SL. Effects of decomposition levels of wavelets in image compression algorithms. Journal of Biomedical Sciences 2016; 5(4): 29.

89. Abdulrahman AA, Rasheed M, Shihab S. The role of intermittent wavelets in image analysis. Electronics Science Technology and Application 2020.

90. Abdulrahman AA, Rasheed M, Shihab S. Various techniques for De noise image. Electronics Science Technology and Application 2020.

91. Rasheed M, Abdulrahman AA, Shihab S. The effect of set partitioning in hierarchical trees with wavelet decomposition levels algorithm for image compression. Electronics Science Technology and Application 2020; 7(3): 40-46.

92. Zadeh LA. Fuzzy sets. Information and Control 1965; 8(3): 338-353. 